\title{
Trastuzumab-associated cardiac events in the Persephone trial
}

\author{
Helena M Earl ${ }^{\star}, 1,2,3$, Anne-Laure Vallier ${ }^{4}$, Janet Dunn ${ }^{5}$, Shrushma Loi ${ }^{5}$, Emma Ogburn $^{5}$, Karen McAdam ${ }^{3,6}$, \\ Luke Hughes-Davies ${ }^{1,3}$, Adrian Harnett ${ }^{7,8}$, Jean Abraham ${ }^{1,2,3}$, Andrew Wardley ${ }^{9}$, David A Cameron ${ }^{10}$, \\ David Miles ${ }^{11}$, loannis Gounaris ${ }^{12,13}$, Chris Plummer ${ }^{14}$ and Louise Hiller ${ }^{5}$
}

Background: We report cardiac events in the Persephone trial which compares 6-12 months of adjuvant trastuzumab in women with confirmed HER2-positive, early-stage breast cancer.

Methods: Clinical cardiac events were defined as any of the following: symptoms and/or signs of congestive heart failure (CHF) and new or altered CHF medication. In addition, left ventricular ejection fraction (LVEF) was measured at baseline and then 3 monthly for 12 months.

Results: A total of 2500 patients, aged 22-82, were included: 1251 randomised to 12 months and 1249 to 6 months of trastuzumab treatment. A total of $93 \%$ (2335/2500) received anthracyclines, 49\% of these (1136/2335) with taxanes. Cardiotoxicity delayed treatment in $6 \%$ of 12 -month and $4 \%$ of 6 -month patients $(P=0.01)$, and stopped treatment early in $8 \%$ $(96 / 1214)$ of 12 -month and $4 \%(45 / 1216)$ of 6 -month patients $(P<0.0001)$. Between 7 and 12 months, more 12 -month than 6 month patients had LVEFs $<50 \%$ ( $8 \%$ vs $5 \% ; P=0.004)$. LVEFs showed quadratic change over time, and 6 -month patients had a more rapid recovery $(P=0.02)$. In a landmark analysis twice as many 12-month patients, free of cardiac events at 6 months, had cardiac problems in months $7-12(6 \%(66 / 1046)$ vs $3 \%(29 / 1035)$ of 6 -month patients $(P=0.0002))$. Lower baseline LVEF predicted more cardiac dysfunction in both arms (reference $\geqslant 65 \%$ : 55 to <65\% OR 1.61 (95\% Cl 1.26-2.04); <55\% OR 5.22 (3.42-7.95)) as did increasing age (reference <50: 50-59 OR 1.58 (1.17-2.12), 60-69 OR 1.91 (1.42-2.57)) 70+ OR 2.72 (1.82-4.08)) and prior use of cardiac medication (OR 8.46 (4.69-15.25)). $>3$ cycles of anthracycline was associated with higher risk of cardiac events only for 12-month patients (OR 1.41 (1.04-1.90)), and not for 6-month patients (OR 1.28 (0.91-1.79)).

Conclusions: We demonstrate significantly fewer cardiac events from 6 months of adjuvant trastuzumab compared with that from 12 months. This cardiac signal adds importance to the question of the optimum duration of adjuvant trastuzumab treatment. If 6 months is proven to have non-inferior outcomes to 12 months treatment, these data would support 6 months as the standard of care.

Trastuzumab has transformed the treatment of HER2-positive breast cancer both in the adjuvant and in the metastatic settings. The results of the US and international adjuvant trastuzumab trials published in 2005-2007 (Hortobagyi, 2005; PiccartGebhart et al, 2005; Romond et al, 2005; Slamon et al, 2011) resulted in a paradigm shift in treatment and established 12 months of adjuvant trastuzumab as the standard of care. Previously HER2-positive breast cancer was associated with poor outcomes. The METABRIC genomic analysis of nearly 2000 breast cancer patients serves as a reminder of the poor prognosis particularly for high-risk ER-negative HER2-positive patients before the introduction of adjuvant trastuzumab (Curtis et al, 2012; Dawson et al, 2013). The HERA trial has reported longterm follow-up results (Goldhirsch A et al, 2013) that have demonstrated a robust improvement in disease-free and overall survival with 12 months of adjuvant trastuzumab but no incremental clinical benefit from extending adjuvant treatment to 24 months.

*Correspondence: Dr HM Earl; E-mail: hme22@cam.ac.uk

Received 29 April 2016; revised 27 September 2016; accepted 3 October 2016; published online 22 November 2016

(c) 2016 Cancer Research UK. All rights reserved 0007 - 0920/16 
Cardiac dysfunction with reduced left ventricular ejection fraction (LVEF) has been recognised as a side effect of trastuzumab therapy since the early days of its use (Seidman et $a l, 2002)$ and has since been reported in all adjuvant trials (Tan-Chiu et al, 2005; Suter et al, 2007; Perez et al, 2008; Procter et al, 2010; Russell et al, 2010; de Azambuja et al, 2014). The pathophysiology of the observed cardiac toxicity is not completely understood. HER2 receptors are present on cardiac myocytes, and, without normal HER2 function, there may be accumulation of reactive oxygen species resulting in dysfunction (Zeglinski et al, 2011). Trastuzumab may also impair mitochondrial function through intracellular downstream events resulting in ATP depletion and therefore contractile dysfunction (Force, Krause, van Etten, 2007). Sequential cardiac stresses from anthracycline administration preceding trastuzumab treatment are also likely to significantly contribute to cardiac problems (Ewer et al, 1999; Ewer et al, 2005). The PHARE trial, which has also looked at reducing trastuzumab duration to 6 months compared with the standard of 12 months (Pivot et al, 2013), demonstrated a reduction in cardiac dysfunction to $4 \%$ in the 6-month arm (Pivot et al, 2015). The cardiac data from the PHARE (Pivot et al, 2015) and HERA (Tan-Chiu et al, 2005; Suter et al, 2007; Procter et al, 2010; de Azambuja et al, 2014) trials and the US studies (Perez et al, 2008; Russell et al, 2010) show a clear relationship between the duration of trastuzumab exposure and incidence of cardiac dysfunction. Notwithstanding this important finding, there are increasing numbers of patients on trastuzumab for long periods of time in the metastatic setting, and in these patients there seems little objective data to indicate a cumulative dose form of toxicity as seen with anthracyclines (Jensen, 2006; Mantarro et al, 2016).

There would be clear advantages in reducing the risk of cardiac dysfunction, if the duration of adjuvant trastuzumab could be shortened to 6 months or less without sacrificing clinical efficacy. From 2007 to the end of July 2015, the Persephone trial randomised HER2-positive patients to either 6- or 12-months of trastuzumab treatment (Earl et al, 2014). We present here the cardiology sub-study with the pre-specified secondary end point of cardiac function in the first 2500 patient cohort. The Persephone trial was designed to 'map onto standard practice' in the UK, and therefore our results will be applicable to 'realworld patients'.

\section{PATIENTS AND METHODS}

Study design. The Persephone trial is a UK phase III randomised non-inferiority trial comparing 6 months of trastuzumab treatment to the standard 12-month duration in women with histologically confirmed HER2-positive early invasive breast cancer receiving a standard chemotherapy regimen in the adjuvant or neo-adjuvant setting. These patients had stage I-IIIa disease. This trial was approved by Multi-Research Ethics Committee (07/MRE08/35), is conducted in accordance with the declaration of Helsinki and supported by the NCRN (No 4078).

The primary end point is disease-free survival (DFS), with cardiac function a secondary end point. The Data and Safety Monitoring Committee reviewed full cardiac data on the first 2500 patients and advised both a reduction in frequency of LVEF measurements from 3-monthly to the new UK standard practice (minimum 4-monthly; Jones et al, 2009) as well as the publication of the sub-study cardiac event data ahead of the primary endpoint of the trial.

These 2500 patients were randomised to receive trastuzumab intravenously (IV) for either 12 months (18 cycles) or 6 months (9 cycles) using a schedule of one loading dose of $8 \mathrm{mg}$ per kilogram of body weight $\left(\mathrm{kg}^{-1}\right)$ IV followed by 3-weekly maintenance doses of $6 \mathrm{mg} \mathrm{kg}^{-1} \mathrm{IV}$. Trastuzumab administration could be concurrent with taxane (but not anthracyclines) or sequential.

Participants. Inclusion criteria were as follows: histological diagnosis of invasive breast cancer; no evidence of metastatic disease; known hormone receptor status; overexpression of HER2 receptor; bilateral breast cancers provided one of the tumours overexpresses HER2 receptor; clear indication for neo-adjuvant or adjuvant chemotherapy based on clinical and histopathological features; patient fit to receive neo-adjuvant or adjuvant chemotherapy and trastuzumab in the opinion of the responsible physician; and no previous diagnosis of malignancy unless (i) managed by surgical treatment only and being disease-free for 10 years or (ii) previous basal cell carcinoma, cervical carcinoma in situ or ductal carcinoma in situ of the breast; non-pregnant and non-lactating, with no intention of pregnancy during chemotherapy, and agrees to adopt adequate contraceptive measures if premenopausal and sexually active; no concomitant medical or psychiatric problems that might prevent completion of treatment or follow-up; patients 18 years or older; and written informed consent for the study given at any time before the 10th cycle of trastuzumab. Exclusion criteria were as follows: significant concomitant cardiac disease or other co-morbidity which in the opinion of the responsible physician added to the risks associated with trastuzumab or cytotoxic chemotherapy; inability to comply with protocol requirements; and patients having already received more than nine cycles of trastuzumab before randomisation.

In particular participants should have no significant cardiac disease or co-morbidity that added to the cardiac risks associated with trastuzumab and chemotherapy, in the opinion of the principal investigator (PI). However, Persephone was a trial designed to 'map onto standard practice' and therefore did not exclude any patients who, in the opinion of the PIs at recruiting sites, would otherwise be considered fit enough to receive chemotherapy and the standard of 12 months of adjuvant trastuzumab treatment.

Randomisation and masking. The trial was open label and we randomly assigned patients (1:1) to 12 months or 6 months of trastuzumab treatment using a central computerised minimisation procedure. Stratification by minimisation was done by ER status, chemotherapy type, chemotherapy timing and trastuzumab timing according to the protocol. Randomisation was at any point before cycle 10 of trastuzumab.

Cardiac endpoints. 'Clinical cardiac dysfunction' was defined as any or all of the following: symptoms or signs of CHF or new/ altered cardiac medication prescribed during the 12 months after starting trastuzumab. Patients on trastuzumab were reviewed by the study team on site every 3 weeks, and every 3 months 'clinical cardiac dysfunction' was reported on the case record forms, to the trials office. Patients were questioned about symptoms of CHF and if these were present, examined by a physician for signs of $\mathrm{CHF}$. Other cardiac events included cardiac death, defined as death with at least one cause recorded relating to a cardiac issue. 'Low LVEF' was a cardiac end point, and was defined as LVEF measurement $<50 \%$ at any point, or a report recording systolic function below normal but without LVEF values. LVEF was recorded at the start of trastuzumab (baseline) and at 3-monthly intervals for 12 months in both randomised arms and regardless of the timing of randomisation with respect to start of trastuzumab. Echocardiography (ECHO) or multi-gated acquisition cardiac scanning (MUGA) was undertaken, preferably using the same method throughout for each individual. Although we recognise that these two methods may produce results differing in some aspects, $\mathrm{ECHO}$ and MUGA were treated equivalently in the data analysis. Cardiac 
monitoring did not routinely continue after 12 months, although some later LVEF measurements were available.

Treatment modifications. Patients developing clinical cardiac dysfunction underwent full cardiac assessment. If LVEF fell below $50 \%$, the protocol advised 'holding' trastuzumab and repeating LVEF at 6-week intervals until LVEF returned to $\geqslant 50 \%$. Only two delays for a maximum of 3 months on account of cardiac toxicity were allowed before trastuzumab was discontinued permanently. In the case of New York Heart Association (NYHA) class III/IV heart failure symptoms (breathlessness at rest or on minimal exertion), trastuzumab was permanently discontinued even after resolution of symptoms or normalisation of LVEF with treatment (The Criteria Committee of the New York Heart Association, 1994; Raphael et al, 2007).

Statistical analysis. Randomised treatment arms and assessment periods were compared by the number of patients reporting treatment modification or cardiac end points and course delivered dose intensity (CDDI; Earl et al, 2008) of trastuzumab using $\chi^{2}$ tests with continuity corrections. Patients' baseline LVEF and the relative changes at 6 and 12 months were assessed using Wilcoxon signed rank tests and Wilcoxon rank sum test.

Random effects modelling was applied to patients' LVEF over time, and results presented graphically as the mean patient values over time for each treatment arm as predicted by the model. Further random effects modelling investigated the influence of chemotherapy type, trastuzumab timing and number of anthracycline cycles. The influence of predictive factors for development of cardiac dysfunction (patients with clinical cardiac dysfunction or a low LVEF during the 12-month reporting period) was assessed using logistic regression and presented with odds ratios and $95 \%$ CIs.

Statistical analysis was on an intention-to-treat basis and therefore all patients were analysed within their randomised treatment arms. All reported $P$-values are two-sided. Analysis was undertaken by Warwick Clinical Trials Unit, using SAS statistical software (version 9.3), following the pre-defined trial Statistical Analysis Plan. Persephone is registered with EudraCT (2006-007018-39), ISRCTN (52968807) and ClinicalTrials.gov (NCT00712140).

\section{RESULTS}

Patients. The first 2500 patients were randomised into the Persephone trial between October 2007 and December 2012 (Figure 1); 1251 were randomised to 12 months and 1249 to 6 months of trastuzumab treatment. These subgroups appeared balanced across treatment arms in terms of patient characteristics and prognostic variables (Table 1), and were representative of the whole Persephone patient sample (values for all 4088 patients shown in parenthesis): median age 55 years (56 years); $94 \%$ white (93\%); 61\% post-menopausal (61\%); 67\% ER positive $(69 \%) ; 48 \%$ $\leqslant 2 \mathrm{~cm}$ diameter (49\%); 67\% grade $3(66 \%)$; and $58 \%$ node negative (59\%). During follow-up 59/2500 (2.4\%) of the patients recurred (local or distant) within 12 months of starting trastuzumab.

Chemotherapy, cardiac medication before trastuzumab and timing of randomisation. A total of $93 \%(2335 / 2500)$ of patients received an anthracycline as part of either anthracycline-based (48\% (1199/2500)) or anthracycline plus taxane-based (45\% (1136/ 2500)) chemotherapy. In all, $65 \%$ received trastuzumab sequentially to chemotherapy and $35 \%$ concomitantly with the nonanthracycline component. Full chemotherapy details are shown in Tables 1 and 2. Only 2\% (50/2500) of patients, balanced across randomised treatment arms, reported being on cardiac medication before start of trastuzumab (Table 1). Randomisation was before the start of trastuzumab or at any time before the 10th cycle. In all, $55 \%(1365 / 2500)$ were randomised up-front, and 82\% (2039/2500) before cycle 4 (Supplementary Table 1), balanced across randomised treatment arms.

Trastuzumab modifications. Complete trastuzumab treatment data are available on 2433 (97\%) of the 2500 patients (Figure 1). Of those randomised to the 12-month arm, 85 (7\%) received 1-9 cycles and 1129 (93\%) received $>10$ cycles of trastuzumab (Figure 1 and Supplementary Figure 1). Of those randomised to the 6-month arm, 1165 (96\%) received 1-9 cycles and 51 (4\%) received $>10$ cycles of trastuzumab. Three patients received no trastuzumab within the trial due to the diagnosis of metastatic disease. Cardiotoxicity was the most common reason for stopping treatment early $(n=141$; Supplementary Figure 1$)$, followed by patient request $(n=76)$. Cardiotoxicity was also given as the

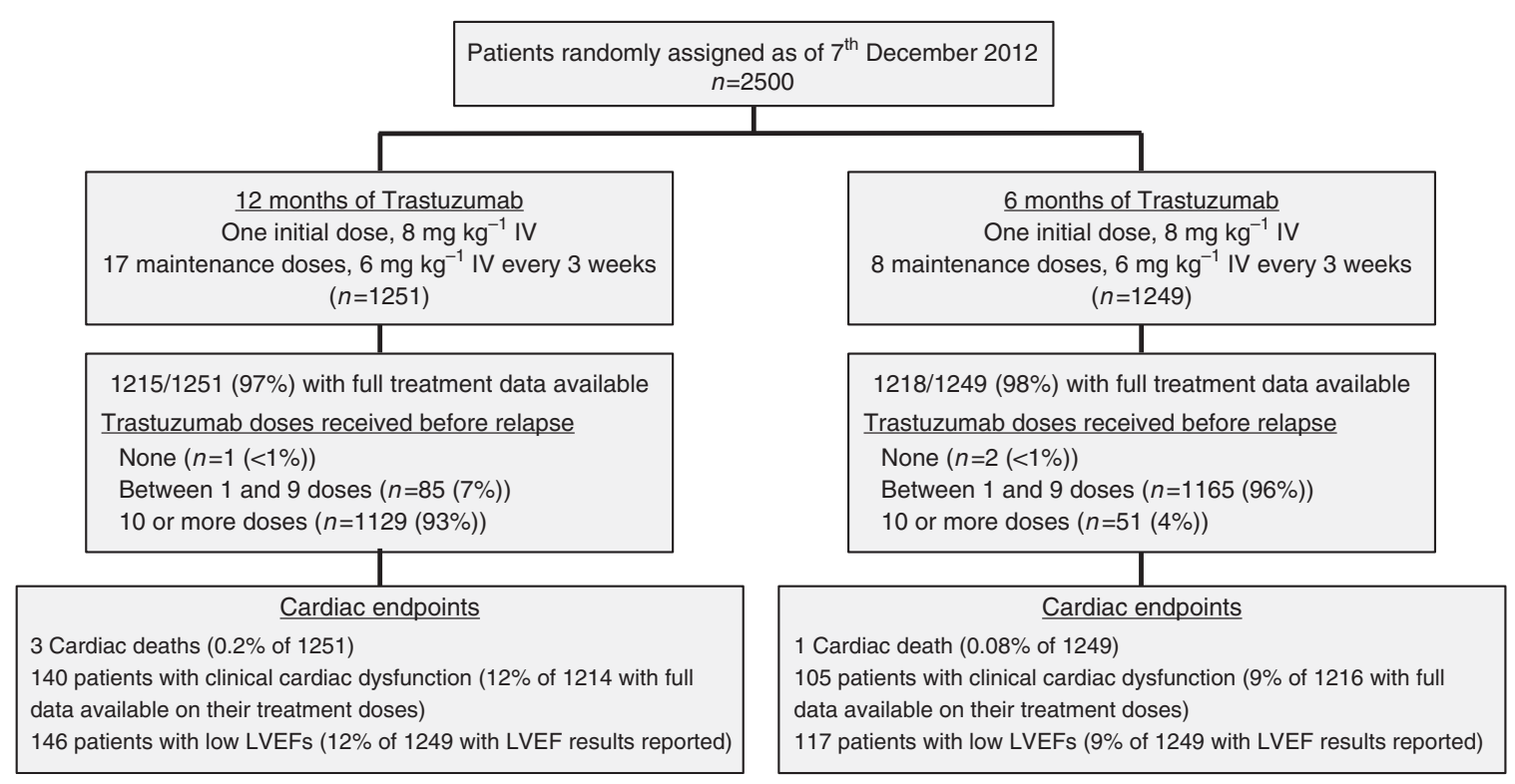

Figure 1. Flow chart of cardiac follow-up and events. 
Table 1. Patient, tumour and treatment details of the first 2500 Persephone patients

\begin{tabular}{|c|c|c|c|}
\hline & $\begin{array}{c}\text { 12-months patients } \\
N(\%)\end{array}$ & $\begin{array}{c}\text { 6-months patients } \\
N(\%)\end{array}$ & $\begin{array}{l}\text { Total } \\
N(\%)\end{array}$ \\
\hline \multicolumn{4}{|l|}{ Patient details } \\
\hline \multicolumn{4}{|l|}{ Age at diagnosis } \\
\hline$<35$ years old & $30(2)$ & $30(2)$ & $60(2)$ \\
\hline $35-49$ years old & $372(30)$ & $358(29)$ & $730(29)$ \\
\hline $50-59$ years old & $400(32)$ & $425(34)$ & $825(33)$ \\
\hline \multicolumn{4}{|l|}{ Ethnicity (2176 (87\%) known) } \\
\hline White & $1021(93)$ & 1019 (95) & 2040 (94) \\
\hline Asian & $31(3)$ & $23(2)$ & $54(2)$ \\
\hline Black & $36(3)$ & $23(2)$ & $59(3)$ \\
\hline Other & $12(1)$ & $11(1)$ & $23(1)$ \\
\hline \multicolumn{4}{|l|}{ BMI (2471 (99\%) known) } \\
\hline High & $838(68)$ & $851(69)$ & $1689(68)$ \\
\hline Post & $670(60)$ & $658(61)$ & $1328(61)$ \\
\hline \multicolumn{4}{|l|}{ Tumour details } \\
\hline \multicolumn{4}{|l|}{ ER status } \\
\hline Negative & $418(33)$ & $416(33)$ & $834(33)$ \\
\hline Positive & $833(67)$ & $833(67)$ & $1666(67)$ \\
\hline \multicolumn{4}{|c|}{ Tumour size of largest invasive tumour at surgery (2319 $(93 \%)$ known) } \\
\hline$\leqslant 2 \mathrm{~cm}$ & $560(48)$ & $560(48)$ & $1120(48)$ \\
\hline$>2$ and $\leqslant 5 \mathrm{~cm}$ & $529(46)$ & $540(47)$ & $1069(46)$ \\
\hline$>5 \mathrm{~cm}$ & $67(6)$ & $63(5)$ & $130(6)$ \\
\hline \multicolumn{4}{|c|}{ Tumour Grade (2410 (96\%) known) } \\
\hline I (well diff.) & $18(2)$ & $22(2)$ & $40(2)$ \\
\hline II (mod. diff.) & $352(30)$ & $360(31)$ & $712(31)$ \\
\hline III (poor diff.) & $783(68)$ & $777(67)$ & $1560(67)$ \\
\hline \multicolumn{4}{|c|}{ Nodal Status at surgery (2483 (99\%) known) } \\
\hline \multicolumn{4}{|l|}{ Chemotherapy type } \\
\hline Anthracycline + taxane based & $568(45)$ & $568(46)$ & $1136(46)$ \\
\hline No taxane and no anthracycline & $2(<1)$ & $2(<1)$ & $4(<1)$ \\
\hline \multicolumn{4}{|l|}{ Chemotherapy timing } \\
\hline Adjuvant & $1093(87)$ & $1092(87)$ & $2185(87)$ \\
\hline Neo-adjuvant & $158(13)$ & $157(13)$ & $315(13)$ \\
\hline \multicolumn{4}{|l|}{ Trastuzumab timing } \\
\hline Concurrent & $442(35)$ & $441(35)$ & $883(35)$ \\
\hline Sequential & $809(65)$ & $808(65)$ & $1617(65)$ \\
\hline \multicolumn{4}{|c|}{ Reported prior use of cardiac medication } \\
\hline Yes & $26(2)$ & $24(2)$ & $50(2)$ \\
\hline No & $1225(98)$ & $1225(98)$ & 2450 (98) \\
\hline
\end{tabular}

reason for more trastuzumab delays in 12-month than in 6-month patients $(6 \%$ vs $4 \%$ of patients $(P=0.01$; Table 3$)$. Significantly more 6-month patients $(1066 / 1164(92 \%))$ received at least $85 \%$ CDDI, compared with 12-month patients (963/1142 (84\%); $P<0.0001)$.

Cardiac events. Full clinical cardiac data were available on 2430 patients (97\%). Clinical cardiac dysfunction was more common in 12-month than in 6-month patients (140/1214 (12\%) vs 105/1216 (9\%) respectively, $P=0.02$; Table 4 ). No differences were apparent in the first 6 months of trastuzumab (8\% of 12-month patients, 7\% of 6 -month patients $(P=0.19))$. However, a significant difference appeared during the $7-12$-month period ( $8 \%$ vs $5 \%$, respectively $(P=0.03))$.

A total of 12,147 LVEF measurements (71\% ECHO, $17 \%$ MUGA, 12\% unknown) were reported in 2498 patients. The use of each modality was balanced across randomised treatment arms. MUGAs appear to have a higher rate of abnormal scores (MUGAs $10 \%$ abnormal compared with $4 \%$ for ECHOs, Table 4$)$. During the first 6 months of treatment, $7 \%$ of 12 -month patients and $8 \%$ of 6-month patients recorded low LVEFs $(P=0.76)$. During months $7-12$, this increased to $8 \%$ of 12 -month patients but fell to $5 \%$ of 6-month patients $(P=0.004$ for difference between treatment arms). Similarly, during the first 6 months of treatment, 
Table 2. Chemotherapy type across chemotherapy and trastuzumab timing

\begin{tabular}{|c|c|c|c|c|}
\hline & $\begin{array}{c}\text { Anthracycline } \\
\text { based } \\
N(\%)\end{array}$ & $\begin{array}{c}\text { Taxane } \\
\text { based } \\
N(\%)\end{array}$ & $\begin{array}{c}\text { Anthracycline }+ \text { Taxane } \\
\text { based } \\
N(\%)\end{array}$ & $\begin{array}{c}\text { No taxane, } \\
\text { no anthracycline } \\
N(\%)\end{array}$ \\
\hline \multicolumn{5}{|c|}{ Chemotherapy timing } \\
\hline \multicolumn{5}{|c|}{ Trastuzumab timing } \\
\hline $\begin{array}{l}\text { Concurrent } \\
\text { Sequential }\end{array}$ & $\begin{array}{c}50(6) \\
1149(71)\end{array}$ & $\begin{array}{c}115(13) \\
46(3)\end{array}$ & $\begin{array}{l}718(81) \\
418(25)\end{array}$ & $\begin{array}{l}0(0) \\
4(1)\end{array}$ \\
\hline
\end{tabular}

Table 3. Trastuzumab treatment modifications by the $\mathbf{2 4 3 0}$ patients with complete trastuzumab treatment information and who received trastuzumab

\begin{tabular}{|c|c|c|c|}
\hline & 12-months patients & 6-months patients & $P$-value \\
\hline $\begin{array}{l}\text { Number of patients } \\
\text { Treatment data in the 1st 6-month period } \\
\text { Treatment data in the 2nd 6-month period }\end{array}$ & $\begin{array}{l}1214 \\
1214 \\
1129\end{array}$ & $\begin{array}{c}1216 \\
1216 \\
51^{a}\end{array}$ & \\
\hline $\begin{array}{l}\text { No. of patients reporting delays due to cardiotoxicity } \\
\text { In } 1 \text { st 6-month period } \\
\text { In } 2 \text { nd 6-month period }\end{array}$ & $\begin{array}{c}72(6 \%) \\
40 / 1214(3 \%) \\
33 / 1129(3 \%)\end{array}$ & $\begin{array}{c}45(4 \%) \\
43 / 1216(4 \%) \\
2 / 51^{\mathrm{a}}(4 \%)\end{array}$ & 0.01 \\
\hline
\end{tabular}

equal proportions of patients across randomised treatment arms recorded significant LVEF falls from baseline, with a similar proportion observed in the second 6-months for 12-month patients, but lower for 6-month patients (Table 4).

Four cardiac deaths were reported. One was assessed as noncardiac as at the time of death from liver metastases, the patient had well-controlled NYHA II heart failure. In the other three patients post-mortem examination demonstrated ischaemic heart disease as the cause of death. In two of these three patients, all LVEF measurements during the 12 months of the trial were normal.

Relative change of LVEF at 6 and 12 months. A total of 2420 patients had an LVEF measured at baseline and at least one other timepoint; 1204 12-month and 1216 6-month patients (Supplementary Table 2). The two treatment arms were well matched for baseline LVEF (12-month median 63\% (IQR 59$67 \%$ ), 6-month median 63\% (IQR 60-67\%), $P=0.22$ ). There were statistically significant reductions in LVEF at the 6 and 12 month time point in both treatment arms (all $P<0.0001$ ). Although similar relative changes from baseline to 6 months were seen across treatment arms (12-month median 0.97 (IQR 0.90-1.02), 6-month median 0.97 (IQR $0.90-1.02, P=0.38$ ), 12-month patients showed significantly greater reductions in LVEF by 12 months (12-month median 0.96 (IQR 0.89-1.02), 6-month median 0.97 (IQR 0.91$1.03, P=0.001)$.

Landmark cardiac dysfunction analysis from 6 months. Cardiac data were available from 2081 patients who were free of cardiac events in their first 6 months of treatment. Significantly more 12month patients $(66 / 1046(6 \%))$ than 6-month patients $(29 / 1035$ $(3 \%))$ were recorded to have a cardiac event in the second 6 month period $(P=0.0002)$.

Random effects modelling. A quadratic curve was found to fit the LVEF data, demonstrating that cardiac function recovers post treatment (Figure 2). This was seen for the entire trial population $(P<0.0001)$ and for each randomised arm separately (both $P<0.0001)$. There was a significant difference between treatments in terms of their change over time $(P=0.02)$; quadratic modelling predicts an earlier recovery of cardiac function after treatment completion in 6-month patients. No significant differences were found between chemotherapy types $(P=0.43)$ or trastuzumab timing $(P=0.79)$ in terms of LVEF changes over time (Supplementary Figures $2 \mathrm{~A}$ and $\mathrm{B}$ ).

Predictive factors for cardiac dysfunction. For both 12-month and 6-month patients, lower baseline LVEF increased the odds of cardiac dysfunction (reference > 65\%: 55 to $<65 \%$ OR 1.61 (95\% CI 1.26-2.04) <55\% OR 5.22 (95\% CI 3.42-7.95); Table 5). In addition, increasing age was found to be linked to increasing odds of cardiac dysfunction (reference <50: 50-59 OR 1.58 (95\% CI 1.17-2.12), 60-69 OR 1.91 (95\% CI 1.42-2.57)) $70+$ OR 2.72 (95\% CI 1.82-4.08)). Chemotherapy type, left-sided radiotherapy, BMI and ethnicity did not appear to influence this end point. Receiving $>3$ cycles of anthracycline was associated with increasing odds of developing cardiac dysfunction in the 12-month trastuzumab arm (OR 1.41 (95\% CI 1.04-1.90)), but not the 6-month arm (OR 1.28 (95\% CI 0.91-1.79)). Further random effects modelling showed that $>3$ compared with $\leqslant 3$ cycles of anthracycline showed a non-significant trend to lower LVEF and slower recovery in the 12 -month $(P=0.19)$ but not in the 6-month arm $(P=0.70$; Supplementary Figure $3 \mathrm{~A}$ and $3 \mathrm{~B})$. In the small number of patients who were taking cardiac medication before starting trastuzumab (25 in the 12-month arm and 23 in the 6-month arm), both arms were at significantly increased risk of developing cardiac dysfunction; 12-month patients had higher risk than 6-month patients (OR 12.47 (95\% CI 5.14-30.25) vs 5.88 (95\% CI 2.55-13.54), respectively).

\section{DISCUSSION}

Trastuzumab is a highly effective adjuvant treatment for HER2positive early breast cancer given in addition to chemotherapy. However, cardiotoxicity has been demonstrated consistently in patients receiving trastuzumab. In 2500 patients in the Persephone trial, we compared cardiotoxicity between the 6- and 12-month arms. Significantly more clinical cardiac dysfunction was seen in 


\begin{tabular}{|c|c|c|c|c|c|c|}
\hline & \multicolumn{3}{|c|}{ 12-months patients } & \multicolumn{3}{|c|}{ 6-months patients } \\
\hline $\begin{array}{l}\text { PERSEPHONE defined cardiac end points } \\
\text { Clinical cardiac dysfunction } \\
\text { Symptoms of } \mathrm{CHF}^{\mathrm{c}} \\
\text { Signs of CHF } \\
\text { New medication for cardiac disease } \\
\text { Low } \text { LVEF }^{\mathbf{d}} \\
\text { Cardiac death }\end{array}$ & $\begin{array}{c}140 / 1214(12 \%) \\
78 \\
27 \\
97 \\
146 / 1249(12 \%) \\
3 / 1251 \\
\end{array}$ & $\begin{array}{c}102 / 1214(8 \%) \\
55 \\
21 \\
65 \\
91 / 1249(7 \%) \\
0 / 1251 \\
\end{array}$ & $\begin{array}{c}91 / 1196(8 \%) \\
41 \\
12 \\
72 \\
101 / 1201(8 \%) \\
0 / 1234^{f} \\
\end{array}$ & $\begin{array}{c}105 / 1216(9 \%) \\
53 \\
17 \\
75 \\
117 / 1249(9 \%) \\
1 / 1249 \\
\end{array}$ & $\begin{array}{c}84 / 1216(7 \%) \\
41 \\
15 \\
62 \\
96 / 1249(8 \%) \\
0 / 1249 \\
\end{array}$ & $\begin{array}{c}62 / 1174(5 \%) \\
22 \\
7 \\
52 \\
59 / 1114(5 \%) \\
0 / 1230^{f}\end{array}$ \\
\hline $\begin{array}{l}\text { Significant LVEF falls } \\
\text { An absolute decrease from baseline of } \geqslant 10 \% \text { to } \\
\text { below } 50 \% \\
\text { An LVEF }<50 \% \text { after a baseline of } \geqslant 59 \%\end{array}$ & 106/1204 (9\%) & 63/1198 (5\%) & 68/1162 (6\%) & 89/1216 (7\%) & $68 / 1214(6 \%)$ & 43/1086 (4\%) \\
\hline \multicolumn{7}{|l|}{ Number of LVEF tests reported as low } \\
\hline \multicolumn{7}{|c|}{ 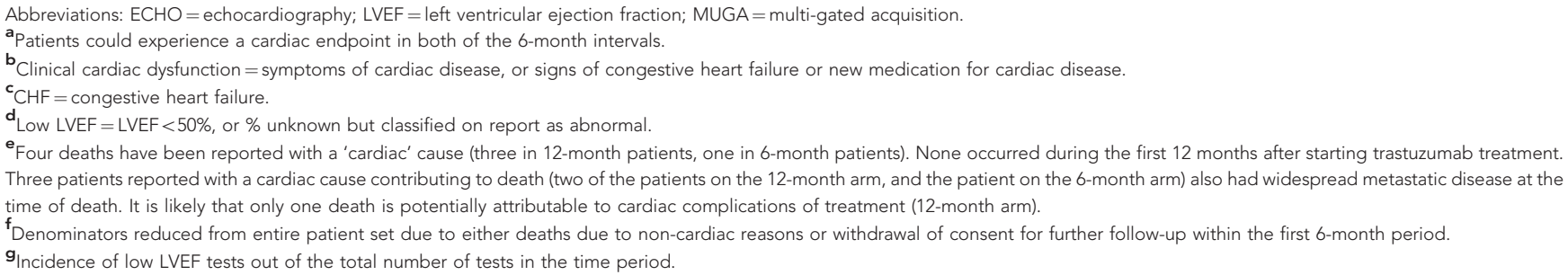 } \\
\hline
\end{tabular}

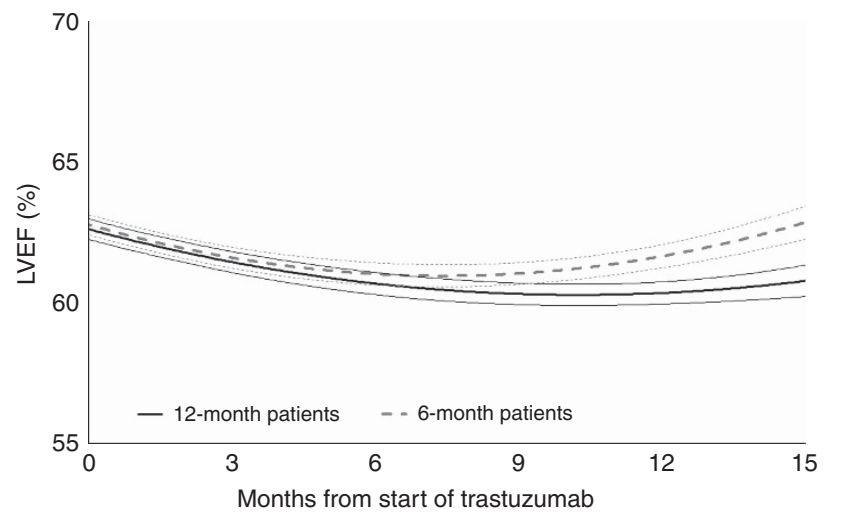

Figure 2. Random Effects modelling predicted lines and 95\% Cls, split by randomised treatment arm.

12-month patients compared with that in 6-month patients (13\% vs $10 \% ; P=0.04$ ), and twice as many 12 -month as 6 -month patients stopped early because of cardiac events ( $8 \%$ vs $4 \%$ : $P<0.0001)$. Consequently, fewer 12 -month than 6-month patients received CDDI of $>85 \%$ ( $84 \%$ vs $92 \%: P<0.0001)$. These data are similar to those reported from the PHARE trial (Pivot et al, 2015). In addition, the HERA trial (Procter et al, 2010) has reported an increased number of patients who developed clinical cardiac dysfunction and reduced LVEF measurements in the 24-month trastuzumab arm. As 24-month treatment has not been shown to be more effective than the standard of 12 months and has caused more cardiotoxicity, 12 months treatment remains the standard at present.

Could the risk of cardiac dysfunction from trastuzumab be decreased by reducing the length of adjuvant treatment? In the Persephone trial the principal research question concerns the non- inferiority for DFS of 6 months compared with the standard of 12 months. In accordance with the trial statistical analysis plan, the primary endpoint will be analysed in late 2017. If 6-month treatment proves to be non-inferior to 12 -month treatment in terms of DFS, then the reduced cardiac problems reported for 6 months of trastuzumab treatment will provide an additional reason to adopt 6 months in place of the standard 12 months of treatment.

Our modelling of the LVEF measurements on the whole population shows some interesting comparisons between the 6- and 12-month arms. Both appear to fit best into a quadratic model during treatment and on recovery. For the 12-month arm, the LVEF data show a lower LVEF nadir and a slower recovery than for the 6-month arm. This suggests that the longer the trastuzumab treatment, the greater the cardiac dysfunction and the longer the recovery time. This effect may not be limited to those with LVEF falls to $<50 \%$ and could potentially result in long-term susceptibility to adverse cardiac events because of a longer, more marked period of myocardial stress. These findings would be another reason to reduce adjuvant trastuzumab to 6 months if this length of treatment proves non-inferior to 12 months.

The methods used to measure LVEF highlighted some interesting issues. ECHO was more frequently used $(71 \%$ of cases) than MUGA (17\% of cases); however, MUGAs had $10 \%$ abnormal values compared with only $4 \%$ for ECHOs. It is possible that MUGAs are more sensitive than ECHOs, or alternatively that MUGAs were the 'preferred' modality when cardiac problems were suspected on clinical grounds. Moreover in some hospitals the normal range of LVEF measured by MUGA is lower (e.g. $>43 \%)$ than that for ECHO $(\geqslant 55 \%)$, which means that it is more likely that a MUGA result will be $<50 \%$. In our analysis, following National Guidelines we took a cut-off of $50 \%$ regardless of modality of assessment, and this is likely to have influenced the incidence of 'abnormal' results for the MUGA investigations. 
Table 5. Predictive factors for development of cardiac dysfunction, by randomised treatment arm

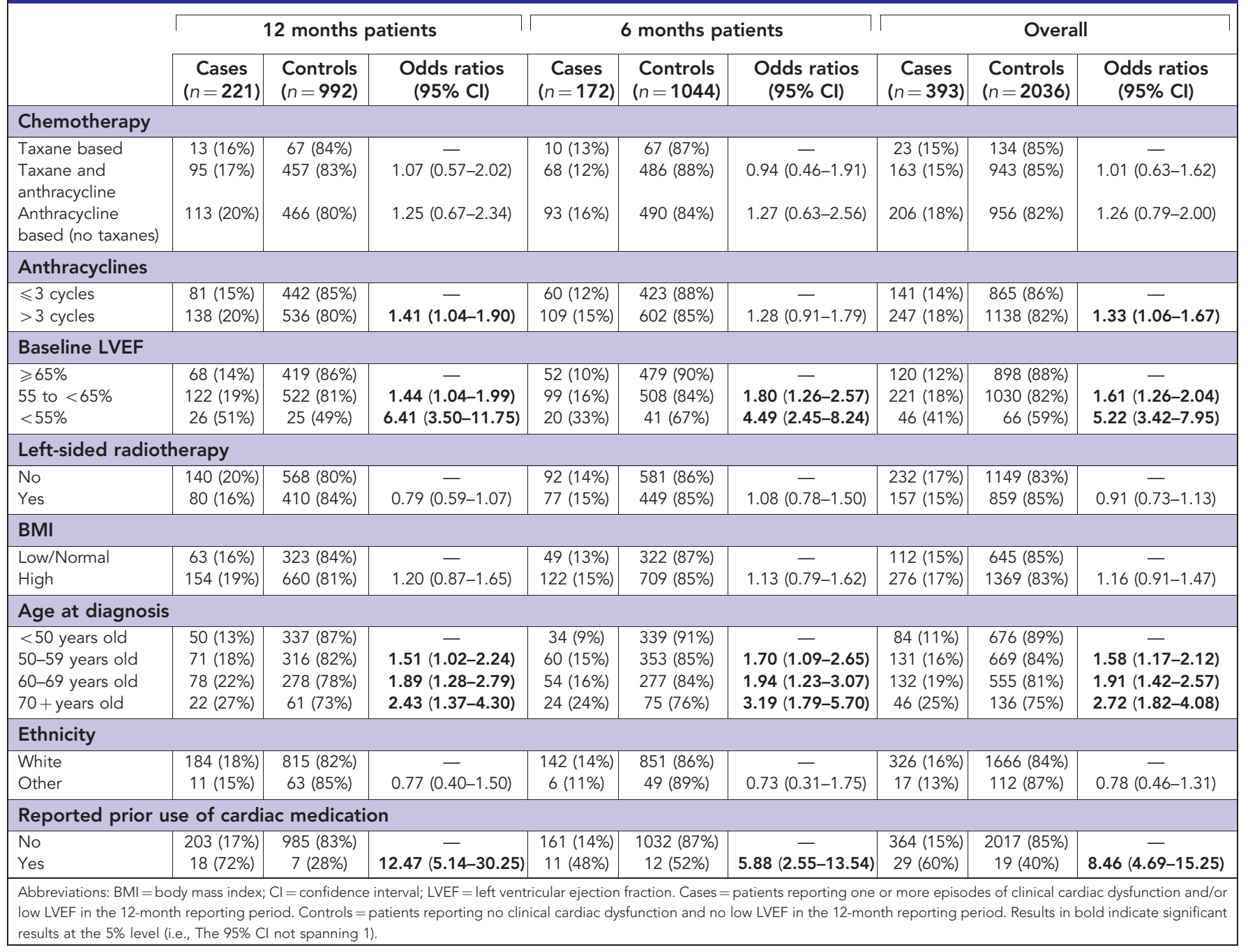

Some researchers have reported genetic alterations within the HER2 receptor that may predispose to cardiac toxicity from trastuzumab (Beauclair et al, 2007; Halyard et al, 2009; Ng et al, 2014). In a companion translational pharmaco-genetic research study (Persephone PGSNPs), a blood sample collection is in progress, which will allow us not only to validate these potential predictive genetic alterations but also in a genome-wide association study to discover other possible genetic predisposition factors for trastuzumab-induced cardiotoxicity.

Our study shows that regardless of 6- or 12-month therapy, both age and baseline LVEF are important predictive factors for cardiac dysfunction. As a surrogate for radiotherapy effects, we compared patients who had right with those with left-sided tumours. Despite other reports of increased cardiotoxicity after radiotherapy for leftsided HER2-positive breast cancer (Roca et al, 2013), we did not find an interaction with radiotherapy and sidedness, although the followup is relatively short. In terms of age, comparing patients under 50 years with those in the next two decades and those over 70 years shows an increasing risk with age. Those over 70 years have an odds ratio of 2.72 for cardiac dysfunction, which would suggest that the predicted benefit of trastuzumab in this age group would have to be significant to balance the risk of cardiac dysfunction. In terms of lower baseline LVEF, patients with LVEF between 50 and 55\% have an odds ratio of 5.22 (95\% CI 3.42-7.95) of developing cardiac dysfunction with low LVEF measurements when compared to a reference of $\geqslant 65 \%$. However, these data should be interpreted cautiously because if a patient is at baseline close to the lower limit of normal LVEF value (50\%), then even a small absolute \% LVEF fall, possibly not clinically meaningful, would take them below this normal threshold. Anthracyclines have been demonstrated to significantly compound trastuzumab cardiotoxicity, nowhere more elegantly that in the BCIRG trial (Slamon et al, 2011) which excluded anthracyclines in one experimental arm. In many studies this interactive effect is significantly greater when more than three cycles of anthracycline are given. Our study is the first to observe a differential effect between 6 and 12 months of trastuzumab therapy, with more than three cycles of anthracycline predicting a higher risk of cardiac toxicity in the 12-month but 'not' in the 6-month arm (OR 1.41 (95\% CI 1.04-1.90) and 1.28 (0.91-1.79), respectively). Although Slamon et al. have shown that anthracyclines can be substituted by carboplatin and long-term outcomes are not significantly different, nevertheless anthracyclines are highly effective chemotherapeutic agents (Poole et al, 2006) and are still received by the majority of breast cancer patients in the adjuvant setting. In addition CEP17 and topo-isomerase II amplification (more common in the HER2positive population) have been reported in a meta-analysis to be predictive biomarkers of anthracycline benefit (Bartlett et al, 2015). Controversy remains with regard to discontinuing the use of anthracyclines altogether in HER2-positive disease, and therefore we should endeavour to develop guidance to maximise the cardiac safety of the anthracycline/trastuzumab combination.

Often clinical trials exclude patients with common risk factors. This can limit the general applicability of their results, and significant genetic differences have been demonstrated between 
patients in clinical trials and those included in the Cancer Genome Atlas (Geifman and Butte, 2016). It is important to emphasise that the Persephone trial was designed to 'map on to standard NHS practice' in the 'real world': The patients included in the trial have the same cardiac risk profile as those receiving standard chemotherapy followed by 12 months of trastuzumab treatment. This has important implications for the interpretation and applicability of the results of this cardiology sub-study and of the future non-inferiority comparison of efficacy.

In conclusion, in the 2500 patient Persephone cardiology substudy, patients on the 6-month arm had significantly fewer cardiac events than on the 12-month arm, particularly if they had received $>3$ cycles of anthracyclines.

\section{ACKNOWLEDGEMENTS}

The trial was funded by the National Institute of Health Research (NIHR) Health Technology Assessment Programme (HTA), funding project grant number 06/303/98. We acknowledge the 382 investigators and their teams from 158 participating UK centres who entered patients into the Persephone trial. Our gratitude also goes to the 2500 women who kindly participated in our study. We thank the Data Safety and Monitoring Committee members: Richard Bell (Deakin University, Geelong, Australia), Roger A'Hern (Institute of Cancer Research - Clinical Trials Statistical Unit, Division of Clinical Studies, Royal Marsden NHS Foundation Trust, Sutton, Surrey, UK) and Marianne Nicolson (Anchor Unit, Aberdeen Royal infirmary, Aberdeen, UK), who have provided professional advice throughout the study, and encouraged us to analyse and report data on cardiac dysfunction in the first 2500 patients. We thank the trials unit staff: Phase 3 coordination at Warwick Clinical Trials Unit, Coventry, UK; translational coordination at University of Cambridge, Addenbrookes Hospital, Cambridge, UK; and statistical analysis at Warwick Clinical Trials Unit, Coventry, UK.

\section{CONFLICT OF INTEREST}

HME reports grants from NIHR HTA during the conduct of the study. JD reports grants from NIHR HTA during the conduct of the study. SL reports grants from NIHR HTA during the conduct of the study. LH reports grants from NIHR HTA during the conduct of the study. AW reports personal fees from Roche, outside the submitted work. DC reports his institution was paid for consultancy work by DC from Roche, outside the submitted work. A-LV, EO, AH, IG, CP, DM, LH-D, KM and JH declare no conflicts of interest.

\section{REFERENCES}

Bartlett JMS, McConkey C, Munro A, Desmedt C, Dunn J, Larsimont D, O'Malley F, Cameron DA, Earl HM, Poole CJ, Shepherd LE, Cardoso F, Jensen MB, Caldas C, Twelves CJ, Rea DW, Ejlertsen B, Pritchard KI, Di Leo A. for the HER2/TII $\alpha$ meta-analysis study group (2015) Predicting anthracycline benefit: TOP2A and CEP17-not only but also. J Clin Oncol 33: $1680-1687$.

Beauclair S, Formento P, Fischel JL, Lescaut W, Largillier R, Chamorey E, Hofman P, Ferrero JM, Pagès G, Milano G (2007) Role of the HER2 [Ile655Val] genetic polymorphism in tumorogenesis and in the risk of trastuzumab-related cardiotoxicity. Ann Oncol 18: 1335-1341.

Curtis C, Shah SP, Chin SF, Turashvili G, Rueda OM, Dunning MJ, Speed D, Lynch AG, Samarajiwa S, Yuan Y, Gräf S, Ha G, Haffari G, Bashashati A, Russell R, McKinney S. METABRIC GroupLangerød A, Green A, Provenzano E, Wishart G, Pinder S, Watson P, Markowetz F, Murphy L, Ellis I, Purushotham A, Børresen-Dale AL, Brenton JD, Tavaré S,
Caldas C, Aparicio S (2012) The genomic and transcriptomic architecture of 2,000 breast tumours reveals novel subgroups. Nature 486:

346-352.

Dawson SJ, Rueda OM, Aparicio S, Caldas C (2013) A new genome-driven integrated classification of breast cancer and its implications. EMBO J 32: 617-628.

de Azambuja E, Procter MJ, van Veldhuisen DJ, Agbor-Tarh D, Metzger-Filho O, Steinseifer J, Untch M, Smith IE, Gianni L, Baselga J, Jackisch C, Cameron DA, Bell R, Leyland-Jones B, Dowsett M, Gelber RD, Piccart-Gebhart MJ, Suter TM (2014) Trastuzumab-associated cardiac events at 8 years of median follow-up in the Herceptin Adjuvant trial (BIG 1-01). J Clin Oncol 32: 2159-2165.

Earl HM, Cameron DA, Miles D, Wardley AM, Ogburn E, Vallier A-L, Loi S, Hiller L, Dunn J (2014) Persephone: duration of trastuzumab with chemotherapy in patients with HER2 positive early breast cancer: six versus twelve months. J Clin Oncol 32(Suppl 15): abstract TPS656.

Earl HM, Hiller L, Dunn JA, Bathers S, Harvey P, Stanley A, Grieve RJ, Agrawal RK, Fernando IN, Brunt AM, McAdam K, O’Reilly S, Rea DW, Spooner D. CJ Poole on behalf of the NEAT Investigators (2008) NEAT: National Epirubicin Adjuvant Trial -toxicity, delivered dose intensity and quality of life. Br J Cancer 99: 1226-1231.

Ewer MS, Gibbs HR, Swafford J, Benjamin RS (1999) Cardiotoxicity in patients receiving trastuzumab (Herceptin): primary toxicity, synergistic or sequential stress, or surveillance artifact? Semin Oncol 26(Suppl 12): 96-101.

Ewer MS, Vooletich MT, Durand JB, Woods ML, Davis JR, Valero V, Lenihan DJ (2005) Reversibility of trastuzumab-related cardiotoxicity: new insights based on clinical course and response to medical treatment. $J$ Clin Oncol 23: 7820-7826.

Force T, Krause DS, van Etten A (2007) Molecular mechanisms of cardiotoxicity in tyrosine kinase inhibition. Nat Rev Med 7: 332-344.

Geifman N, Butte AJ (2016) Do cancer clinical trial populations truly represent cancer patients? A comparison of open clinical trials to the Cancer Genome Atlas. Pac Symp Biocomput 21: 309-320.

Goldhirsch A, Gelber RD, Piccart-Gebhart MJ, de Azambuja E, Procter M, Suter TM, Jackisch C, Cameron D, Weber HA, Heinzmann D, Dal Lago L, McFadden E, Dowsett M, Untch M, Gianni L, Bell R, Köhne CH, Vindevoghel A, Andersson M, Brunt AM, Otero-Reyes D, Song S, Smith I, Leyland-Jones B, Baselga J. Herceptin Adjuvant (HERA) Trial Study Team (2013) 2 years versus 1 year of adjuvant trastuzumab for HER2-positive breast cancer (HERA): an open-label, randomised controlled trial. Lancet 382: 1021-1028.

Halyard MY, Pisansky TM, Dueck AC, Suman V, Pierce L, Solin L, Marks L, Davidson N, Martino S, Kaufman P, Kutteh L, Dakhil SR, Perez EA (2009) Radiotherapy and adjuvant trastuzumab in operable breast cancer: tolerability and adverse event data from the NCCTG Phase III Trial N9831. J Clin Oncol 27: 2638-2644.

Hortobagyi GN (2005) Trastuzumab in the treatment of breast cancer. N Engl J Med 353: 1734-1736.

Jensen BV (2006) Cardiotoxic consequences of anthracycline-containing therapy in patients with breast cancer. Semin Oncol 33(Suppl 8): 15-21.

Jones AL, Barlow M, Barrett-Lee PJ, Canney PA, Gilmour IM, Robb SD, Plummer CJ, Wardley AM, Verrill MW (2009) Management of cardiac health in trastuzumab-treated patients with breast cancer: updated United Kingdom National Cancer Research Institute recommendations for monitoring. $\mathrm{Br} J$ Cancer 100: 684-692.

Mantarro S, Rossi M, Bonifazi M, D’Amico R, Blandizzi C, La Vecchia C, Negri E, Moja L (2016) Risk of severe cardiotoxicity following treatment with trastuzumab: a meta-analysis of randomized and cohort studies of 29000 women with breast cancer. Intern Emerg Med 11: 123-140.

Ng T, Chan M, Khor CC, Ho HK, Chan A (2014) The genetic variants underlying breast cancer treatment-induced chronic and late toxicities: a systematic review. Cancer Treat Rev 40: 1199-1214.

Perez EA, Suman VJ, Davidson NE, Sledge GW, Kaufman PA, Hudis CA, Martino S, Gralow JR, Dakhil SR, Ingle JN, Winer EP, Gelmon KA, Gersh BJ, Jaffe AS, Rodeheffer RJ (2008) Cardiac safety analysis of doxorubicin and cyclophosphamide followed by paclitaxel with or without trastuzumab in the North Central Cancer Treatment Group N9831 adjuvant breast cancer trial. J Clin Oncol 26: 1231-1238.

Piccart-Gebhart MJ, Procter M, Leyland-Jones B, Goldhirsch A, Untch M, Smith I, Gianni L, Baselga J, Bell R, Jackisch C, Cameron D, Dowsett M, Barrios CH, Steger G, Huang CS, Andersson M, Inbar M, Lichinitser M, Láng I, Nitz U, Iwata H, Thomssen C, Lohrisch C, Suter TM, Rüschoff J, 
Suto T, Greatorex V, Ward C, Straehle C, McFadden E, Dolci MS, Gelber RD. Herceptin Adjuvant (HERA) Trial Study Team (2005) Trastuzumab after adjuvant chemotherapy in HER2-positive breast cancer. N Engl J Med 353: 1659-1672.

Pivot X, Romieu G, Debled M, Pierga JY, Kerbrat P, Bachelot T, Lortholary A, Espié M, Fumoleau P, Serin D, Jacquin JP, Jouannaud C, Rios M, Abadie-Lacourtoisie S, Tubiana-Mathieu N, Cany L, Catala S, Khayat D, Pauporté I, Kramar A. PHARE trial investigators (2013) 6 months versus 12 months of adjuvant trastuzumab for patients with HER2-positive early breast cancer (PHARE): a randomised phase 3 trial. Lancet Oncol 14: 741-748.

Pivot X, Suter T, Nabholtz JM, Pierga JY, Espie M, Lortholary A, Khayat D, Pauporte I, Romieu G, Kramar A, Fumoleau P (2015) Cardiac toxicity events in the PHARE trial, an adjuvant trastuzumab randomised phase III study. Eur J Cancer 51: 1660-1666.

Poole CJ, Earl HM, Hiller L, Dunn JA, Bathers S, Grieve RJ, Spooner DA, Agrawal RK, Fernando IN, Brunt AM, O’Reilly SM, Crawford SM, Rea DW, Simmonds P, Mansi JL, Stanley A, Harvey P, McAdam K, Foster L, Leonard RC, Twelves CJ. NEAT Investigators and the SCTBG (2006) Epirubicin plus cyclophosphamide, methotrexate, and 5-Fluorouracil in the adjuvant treatment of early breast cancer. NEJM 355: 1851-1862.

Procter M, Suter TM, de Azambuja E, Dafni U, van Dooren V, Muehlbauer S, Climent MA, Rechberger E, Liu WT, Toi M, Coombes RC, Dodwell D, Pagani O, Madrid J, Hall M, Chen SC, Focan C, Muschol M, van Veldhuisen DJ, Piccart-Gebhart MJ (2010) Longer-term assessment of trastuzumab-related cardiac adverse events in the Herceptin Adjuvant (HERA) trial. J Clin Oncol 28: 3422-3428.

Raphael C, Briscoe C, Davies J, Whinnett IZ, Manisty C, Sutton R, Mayet J, Francis DP (2007) Limitations of the New York Heart Association functional classification system and self-reported walking distances in chronic heart failure. Heart 93: 476-482.

Roca L, Diéras V, Roché H, Lappartient E, Kerbrat P, Cany L, Chieze S, Canon JL, Spielmann M, Penault-Llorca F, Martin AL, Mesleard C, Lemonnier J, de Cremoux P (2013) Correlation of HER2, FCGR2A, and FCGR3A gene polymorphisms with trastuzumab related cardiac toxicity and efficacy in a subgroup of patients from UNICANCER-PACS 04 trial. Breast Cancer Res Treat 139: 789-800.

Romond EH, Perez EA, Bryant J, Suman VJ, Geyer Jr CE, Davidson NE, Tan-Chiu E, Martino S, Paik S, Kaufman PA, Swain SM, Pisansky TM, Fehrenbacher L, Kutteh LA, Vogel VG, Visscher DW, Yothers G, Jenkins RB, Brown AM, Dakhil SR, Mamounas EP, Lingle WL, Klein PM, Ingle JN, Wolmark N (2005) Trastuzumab plus adjuvant chemotherapy for operable HER2-positive breast cancer. N Engl J Med 353: $1673-1684$.

Russell SD, Blackwell KL, Lawrence J, Pippen Jr JE, Roe MT, Wood F, Paton V, Holmgren E, Mahaffey KW (2010) Independent adjudication of symptomatic heart failure with the use of doxorubicin and cyclophosphamide followed by trastuzumab adjuvant therapy: a combined review of cardiac data from the National Surgical Adjuvant breast and Bowel Project B-31 and the North Central Cancer Treatment Group N9831 clinical trials. J Clin Oncol 28: 3416-3421.

Seidman A, Hudis C, Pierri MK, Shak S, Paton V, Ashby M, Murphy M, Stewart SJ, Keefe D (2002) Cardiac dysfunction in the trastuzumab clinical trials experience. J Clin Oncol 20: 1215-1221.

Slamon D, Eiermann W, Robert N, Pienkowski T, Martin M, Press M, Mackey J, Glaspy J, Chan A, Pawlicki M, Pinter T, Valero V, Liu M-C, Sauter G, von Minckwitz G, Visco F, Bee V, Buyse M, Bendahmane B, Tabah-Fisch I, Lindsay M-A, Riva A. Crown J for the Breast Cancer International Research Group ${ }^{*}$ (2011) Adjuvant trastuzumab in HER2-positive breast cancer. N Engl J Med 365: 1273-1283.

Suter TM, Procter M, van Veldhuisen DJ, van, Muscholl M, Bergh J, Carlomagno C, Perren T, Passalacqua R, Bighin C, Klijn JG, Ageev FT, Hitre E, Groetz J, Iwata H, Knap M, Gnant M, Muehlbauer S, Spence A, Gelber RD, Piccart-Gebhart MJ (2007) Trastuzumab-associated cardiac adverse effects in the herceptin adjuvant trial. J Clin Oncol 25: 3859-3865.

Tan-Chiu E, Yothers G, Romond E, Geyer Jr CE, Ewer M, Keefe D, Shannon RP, Swain SM, Brown A, Fehrenbacher L, Vogel VG, Seay TE, Rastogi P, Mamounas EP, Wolmark N, Bryant J (2005) Assessment of cardiac dysfunction in a randomized trial comparing doxorubicin and cyclophosphamide followed by paclitaxel, with or without trastuzumab as adjuvant therapy in node-positive, human epidermal growth factor receptor 2-overexpressing breast cancer: NSABP B-31. J Clin Oncol 23: 7811-7819.

The Criteria Committee of the New York Heart Association (1994) Nomenclature and Criteria for Diagnosis of Diseases of the Heart and Great Vessels. 9th edn. pp 253-256. Little, Brown \& Co.: Boston.

Zeglinski M, Ludke A, Jassal DS, Singal P (2011) Trastuzumab-induced cardiac dysfunction: a 'dual-hit'. Exp Clin Cardiol 16: 70-74.

This work is published under the standard license to publish agreement. After 12 months the work will become freely available and the license terms will switch to a Creative Commons AttributionNonCommercial-Share Alike 4.0 Unported License.

${ }^{1}$ Department of Oncology, University of Cambridge, (Box 193-R4) Addenbrooke's Hospital, Hills Road, Cambridge CB2 0QQ, UK; ${ }^{2}$ NIHR Cambridge Biomedical Research Centre and Cambridge Experimental Cancer Medicine Centre, Box 277, Hills Road, Cambridge CB2 0QQ, UK; ${ }^{3}$ Cambridge Breast Unit and Cambridge University Hospitals NHS Foundation Trust, Hills Road, Cambridge CB2 0QQ, UK; ${ }^{4}$ Cambridge Clinical Trials Unit - Cancer Theme, Cambridge University NHS Foundation Trust, Box 279 (S4), Hills Road, Cambridge CB2 0QQ, UK; ${ }^{5}$ Warwick Clinical Trials Unit, University of Warwick, Gibbet Hill Road, Coventry CV4 7AL, UK; ${ }^{6}$ Peterborough and Stamford Hospitals NHS Foundation Trust and Cambridge University Hospital NHS Foundation Trust, Peterborough City Hospital, Edith Cavell Campus, Bretton Gate, Peterborough PE3 9GZ, UK; ${ }^{7}$ Norfolk \& Norwich University Hospital, Colney Lane, Norwich NR4 7UY, UK; ${ }^{8}$ James Paget University Hospital, Lowestoft Rd, Gorleston-on-Sea, Great Yarmouth, Norfolk NR31 6LA, UK; ${ }^{9}$ The Christie NHS Foundation Trust, 550 Wilmslow Rd, Manchester M20 4BX, UK; ${ }^{10}$ University of Edinburgh Cancer Research Centre, IGMM, Western General Hospital, Crewe Road South, Edinburgh EH4 2XR, UK; ${ }^{11}$ Mount Vernon Cancer Centre, Rickmansworth Rd, Northwood, London HA6 2RN, UK; ${ }^{12}$ Cancer Research UK Cambridge Institute, Li Ka Shing Centre, University of Cambridge, Robinson Way, Cambridge CB2 ORE, UK; ${ }^{13}$ The Queen Elizabeth Hospital King's Lynn NHS Foundation Trust, Gayton Rd, King's Lynn, Norfolk PE30 4ET, UK and ${ }^{14}$ Department of Cardiology, Freeman Hospital, Freeman Rd, Newcastle upon Tyne, Tyne and Wear NE7 7DN, UK

Supplementary Information accompanies this paper on British Journal of Cancer website (http://www.nature.com/bjc) 